\title{
O.S.P.
}

L'orientation scolaire et professionnelle

$30 / 2$ | 2001

Varia

\section{H., Joas. La créativité de l'agir.}

Paris : Cerf.

Jean-Charles Cau.

\section{(2) OpenEdition \\ Journals}

Édition électronique

URL : http://journals.openedition.org/osp/5234

DOI : 10.4000/osp.5234

ISSN : 2104-3795

Éditeur

Institut national d'étude du travail et d'orientation professionnelle (INETOP)

Référence électronique

Jean-Charles Cau., «H., Joas. La créativité de l'agir. ", L'orientation scolaire et professionnelle [En ligne], 30/2 | 2001, mis en ligne le 21 juillet 2016, consulté le 22 octobre 2020. URL : http://

journals.openedition.org/osp/5234; DOI : https://doi.org/10.4000/osp.5234

Ce document a été généré automatiquement le 22 octobre 2020

(c) Tous droits réservés 


\section{H., Joas. La créativité de l'agir.}

Paris : Cerf.

Jean-Charles Cau.

\section{RÉFÉRENCE}

Paris : Cerf.

1 Hans Joas est professeur de sociologie à l'Université libre de Berlin et enseigne également à l'Université de Chicago. Auteur d'une monographie sur George Herbert Mead, il consacre plusieurs ouvrages au pragmatisme américain. Il est considéré comme l'un des représentants de la théorie sociale en Allemagne.

2 L'objectif de l'ouvrage est de rechercher les raisons expliquant la forme spécifique de la «théorie sociologique de l'action ». L'idée centrale de l'ouvrage repose sur la discussion des deux modèles dominants de "l'action rationnelle " et de " l'action à visée normative " au profit d'un troisième révélant le caractère créatif de l'agir humain. Quand l'acteur possède une conscience forte du sens de l'orientation de son action, il s'agit d'une action rationnelle en valeur (par conviction) ou en finalité (par confrontation rationnelle des moyens et des buts). Lorsque l'acteur s'inscrit dans une démarche d'intégration sociale, la norme constitue une des conditions premières à la réussite de son action.

Certains passages du livre ont été déjà publiés, notamment les sections relatives à la « théorie Wébérienne du charisme et le problème de la créativité » (pp. 52-74), " la constitution du schéma corporel » (pp. 177-195). Quatre chapitres composent l'ouvrage. Le premier (" la naissance de la théorie de l'action ») s'adresse au sociologue et à l'économiste. Le deuxième (" métaphore de la créativité ») relève de la discussion philosophique. Joas en dégage particulièrement "les déductions de Schopenhauer ", héritées de Kant, en rupture avec une tradition européenne précise l'auteur, et concerne le concept de "volonté » associé à celui de "corporéité ", (p. 128). Le troisième chapitre (" situation - corporéité - socialité ») traite de la créativité en référence au corps, ce corps associé au désir d'être acteur. Quant au quatrième chapitre 
(« la démocratie créative »), il offre au lecteur la conception de la créativité qui a pour caractéristique de s'appuyer sur les concepts de Maslow.

Ainsi, pour ce qu'il en est de la "Kreativitiit ", " apparue après la seconde guerre mondiale, concept hérité de la terminologie psychologique américaine ", Joas pense que ce serait une erreur de croire que « la dimension de la créativité dans l'agir humain n'ait joué qu'un rôle mineur au cours des deux derniers siècles ». Le point de départ serait selon l'auteur la tentative de synthèse de Parsons, dans " The Structure of social Action ", qui inspire Habermas dans sa théorie de l'agir communicationnel. En effet Parsons met en oeuvre un cadre de référence centré " sur l'acteur lui-même, sur les fins qu'il poursuit ", " les normes qui jouent un rôle régulateur dans le choix de ses moyens et un rôle dans la constitution de ses buts ». Puis, Hans Joas propose une typologie des formes sous-lesquelles la créativité est apparue. Dans cette histoire des idées, l'auteur nous présente, comme pour remonter aux sources même de la sociologie, les insuffisances du modèle de l'action rationnelle. De Descartes aux pragmatismes américains (de l'école de Chicago), de Weber à Parsons, de Kant à Habermas, de l'héritage de Hegel aux idées de «production et de révolution » chez Marx, Hans Joas souhaite réconcilier les théories de Weber, Durkheim, Tônnies et Simmel avec la thématique de la créativité. Hans Joas révise donc l'histoire des idées en relevant trois postulats implicites des théories de l'action, relatifs au caractère téléologique de l'action, au contrôle corporel de l'acteur et à l'individualité autonome du sujet agissant.

5 Joas rediscute ces postulats (chapitre III) sur lesquels reposent les modèles rationnels et normatifs. À la lumière de la situation, de la corporéité et de la socialité, la critique porte sur le fait que dans les modèles précédents, « le sujet est capable d'agir en fonction d'un but, deuxièmement qu'il maitrise son corps, troisièmement qu'il est autonome relativement à ses semblables et à son environnement ». L'auteur s'appuie sur Luhman et Habermas pour mettre en doute l'apparente évidence « du schéma de la fin et des moyens dans l'interprétation de l'agir collectif organisé ". Ce que les études empiriques mettent en lumière, nous précise l'auteur, ce n'est donc pas seulement le décalage entre le modèle rationnel et la réalité, mais «l'irréasibilité du modèle rationnel ».

Pour parler du " corps propre de l'être humain ", Joas s'appuie sur les phénomènes transitionnels (pp. 174-177) chers à Winnicott qui définit la faculté d'agir, ce " passage du désir à l'instauration d'une fin ", comme la capacité de "maintenir, à la fois séparées et reliées l'une à l'autre, réalité intérieure et réalité extérieure ». La théorie de l'action, conclut l'auteur, est pourtant " condamnée à intégrer la dimension de la corporéité ".

7 Dans le dernier chapitre, conclusif, Hans Joas relève les oppositions de type audaceprudence, acceptation-discrimination, imagination-épreuve du réel. Ici la référence est faite à « la psychologie humaniste » de Maslow pour différents types de créativité qui ont la faculté de ne pas se rejeter l'un l'autre :

- Primaire pour la mise en oeuvre de processus dits primaires, c'est-à-dire liés à la faculté de représentation, du jeu et de l'enthousiasme ;

- Secondaire pour la production rationnelle, la résolution de problèmes techniques, scientifiques, artistiques, difficultés d'ordre pratique ;

- Intégrée quand elle associe « l'ouverture de l'expression de soi à la responsabilité du contrôle de soi ».

8 En conclusion, la créativité est une dimension de tout agir humain. Mêmes des actes hautement créatifs s'appuient sur des gestes routiniers. Il n'y a donc pas d'action sans 
créativité et pas d'action sociale sans référence à un système, c'est-à-dire un ensemble de normes servant à évaluer les actions. Hans Joas nous propose un modèle sociologique englobant la créativité plutôt que de la condamner à une peine résiduelle.

9 Cet ouvrage aurait donc pu s'intituler Sociologie philosophique et corporéité créative de l'action. 